\title{
Ambulatory monitoring derived blood pressure variability and cardiovascular risk factors in elderly hypertensive patients
}

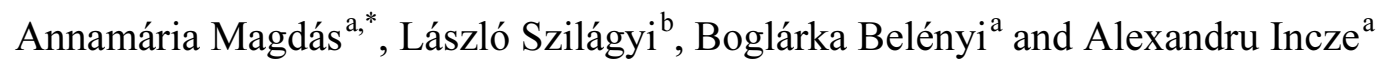 \\ ${ }^{a}$ County Clinical Hospital-Internal Medicine Department IV, University of Medicine and Pharmacy \\ Tîrgu Mureş, Str. Gh. Marinescu Nr. 38, 540139 Tîrgu Mureş, Romania \\ ${ }^{b}$ Faculty of Technical and Human Sciences, Sapientia-Hungarian University of Transylvania, Şos. \\ Sighişoarei 1/C, 540485 Tîrgu Mureş, Romania
}

\begin{abstract}
Hypertension in the elderly is characterized by isolated systolic hypertension and high variability, but its clinical significance is not yet fully understood. The goal of this paper was to assess circadian blood pressure variability (BPV) in elderly hypertensives, and to determine its relationship to cardiovascular risk factors. To achieve this goal, a number of 75 inefficiently treated hypertensive patients were studied, 45 elderly, aged over 60 years, 30 middle-aged, younger than 60 years. After 24-hour ambulatory blood pressure monitoring (ABPM), blood pressure (BP) values, pulse pressure (PP), morning surge were compared between the groups. BPV was calculated using average real variability (ARV). The relationships between BPV, pulse pressure, left ventricular mass index (LVMI), and cardiovascular risk factors were assessed in both groups. As a result, it was found that left ventricular mass ( $\mathrm{p}=0.01)$, PP, morning surge, 24-hour systolic ARV were significantly higher in the elderly group $(\mathrm{p}<0.05)$. In both groups, higher 24-hour BPV was associated with an increase in LVMI. In the elderly population 24-hour BPV was positively correlated to increased PP, total cholesterol and triglyceride levels $(\mathrm{p}<0.05)$. Moreover, it was concluded that ABPM-derived BP variability index could be an early predictive marker of end-organ damage in hypertension. Its reduction might be an important objective of hypertension management in elderly.
\end{abstract}

Keywords: ABPM, blood pressure variability, cardiovascular risk factors, elderly

\section{Introduction}

Due to the global population aging, the incidence of cardiovascular disease is increasing. To reduce financial and human resources, and in order to increase patient compliance to investigation, a personalized out-of-hospital healthcare model is required, which makes possible ambulatory monitoring of the patient with chronic disease.

The cardiovascular system undergoes fluctuation over 24-hour period. An important part of these variations, like changes in heart rate, the occurrence of arrhythmias, as well as circadian blood pressure, can be assessed through ambulatory monitoring [1]. However, in hypertensive patients the

\footnotetext{
*Corresponding author: Annamária Magdás, County Clinical Hospital-Internal Medicine Department IV, University of Medicine and Pharmacy Tîrgu Mureş, Str. Gh. Marinescu Nr. 38, 540139 Tîrgu Mureş, Romania. Tel.: 40-740-962529; Fax: 40-265-206211; E-mail: annamaria.magdas@umftgm.ro.
} 
evaluation of circadian blood pressure (BP) profile is of crucial importance. In the last decade, the potential importance of blood pressure variability (BPV), as an additional cardiovascular (CV) risk factor, captured the attention of researchers [2].

Blood pressure undergoes short-term fluctuations over 24-hour period, which represent the so-called blood pressure variability (BPV) phenomenon, manifested as increased daytime values and decreased nighttime BP values, also known as ,dipping” profile, and an abrupt rise after awakening, also known as morning surge [3]. The difference between the high daytime BP and low nighttime BP depends on baroreflex sensitivity: if the latter decreases, then BP variability rises. Blood pressure variability is a complex phenomenon triggered by humoral, behavioral, central and neural influence. Long-term fluctuations occur over days, weeks, months, seasons and years [4]. Both short-and long-term variability, as well as excessive morning blood pressure surge, is associated with the development of cardiac, vascular, renal organ damage, and with a higher incidence of CV events and mortality [5-8]. In the elderly population, the prevalence of hypertension is higher, compared to middle-aged population, aged below 60 years. Elderly hypertensive subjects display some specific clinical features like isolated systolic hypertension, orthostatic hypotension and higher blood pressure variability [9], due to impaired arterial baroreflex function [10], and increased arterial stiffness, which is associated with morning BP surge pattern [11]. To calculate short-term blood pressure variability, a commonly used parameter is the ambulatory BP monitoring (ABPM) derived 24-hour standard deviation of the mean arterial BP. SD may have its limitations because it reflects only the dispersion of BP measurements around the mean value, and two subjects with different BP measurement sets could display same SD value [12]. This parameter may be sensitive to the low sampling frequency [13]. Therefore to improve the quantification of short-term BPV, a more reliable index was proposed to assess BPV, which also carries prognostic significance, namely the average real variability (ARV). It represents the average of the absolute differences between consecutive SBP measurements over 24 hours [12].

The aim of the study is to evaluate circadian blood pressure profile in inefficiently treated hypertensive elderly patients, to calculate blood pressure variability, and to assess the relationship between 24-hour blood pressure variability and cardiovascular risk factors.

\section{Methods}

This is an observational study. A total of 75 inefficiently treated hypertensive patients were included in the study. Patients were divided into two groups. Forty-five persons, aged $\geq 60$ years, were in the elderly group and 30 subjects $<60$ years, in the middle-aged control group. All subjects gave written informed consent, and the study was approved by local ethic committee, according to the International Ethical Guidelines and Declaration of Helsinki. All patients were admitted to County Hospital, Tîrgu Mureş, Romania. They had a history of hypertension, and were taking medication. Inclusion criteria were: history of hypertension, that is, office $\mathrm{BP} \geq 140 / 90 \mathrm{mmHg}$, ABPM values: awake blood pressure mean below $135 / 85 \mathrm{mmHg}$, or an asleep blood pressure mean below $120 / 70 \mathrm{mmHg}$, according to 2013 ESH/ESC Guidelines for the management of arterial hypertension [14]. Exclusion criteria were pregnant women, type 1 diabetes mellitus, secondary hypertension, angina pectoris, congestive heart failure, arrhythmias, significant valvular disease, and shift workers.

Blood pressure profile was assessed using 24-hour blood pressure monitoring, with a validated device (ABPM05, Meditech, Hungary). Measurements were taken every 15 minutes at daytime (06:00-21:59) and nighttime (22:00-05:59). Blood pressure data (awake, asleep and 24-hour mean BP, 
awake/asleep ratio, pulse pressure, BP surge, 24-hour systolic standard deviation) were edited automatically. Dipper profile was defined as a $>10 \%$ decline in asleep BP mean relative to the awake BP mean. ABPM monitoring began between 8:00-10:00 a.m. BP series were not valid if $>30 \%$ of the measurements were missing. BPV was calculated according to the new formula of read-to-read average real variability (ARV) [12], given as:

$$
A R V=\frac{1}{N-1} \sum_{k=1}^{\mathbf{N}-\mathbf{1}}\left|\mathbf{B P}_{\mathbf{k}+\mathbf{1}}-\mathbf{B P}_{\mathbf{k}}\right|
$$

where $\mathrm{N}$ represents the number of $\mathrm{BP}$ measurements in a given subject, and $\mathrm{BP}_{\mathrm{k}}$ is the blood pressure at measurement number $\mathrm{k}$. Blood samples were obtained, after overnight fasting, to assess cardiovascular risk factors (total cholesterol, triglyceride, blood glucose, creatinine). Body mass index (BMI) was calculated, and glomerular filtration rate (GFR) was appreciated, according to MDRD formula (modification of diet in renal disease study) [15].

To assess left ventricular hypertrophy (LVH), resting electrocardiogram (ECG) and twodimensional (2D) echocardiography, using Hitachi Aloka Prosound 3.5 MHz phased array transducer were performed. Left ventricular mass index (LVMI) was calculated using the Devereux formula [16], corrected for the body surface area.

Data were collected as raw data, using MS Excel program, and compared using Graph Pad Prism version 6 statistical software. Numerical data are represented as mean \pm SD. Means were compared using the t-test for continuous variables and Chi-square test for categorical variables. Correlations were calculated, using Pearson's correlation test. A p value $\leq 0.05$ was considered statistically significant with a confidence interval of $95 \%$.

\subsection{Results}

Demographic, laboratory, ABPM data, BPV defined by average real variability, as well as target organ damage data are shown in Table 1. There was a significant increase in 24-hour mean systolic/diastolic BP, morning BP surge, left ventricular mass, pulse pressure, daytime and 24-hour mean systolic BPV (Figure 1), in the elderly group $(\mathrm{p}<0.05)$. Daytime and nighttime diastolic BP was higher in the control group. BMI and laboratory parameters, the number of dippers/non-dippers was not significantly different between the groups. Glomerular filtration rate estimated with MDRD was significantly lower in the elderly group $(\mathrm{p}=0.03$ ). In both groups 24 -hour mean systolic BP variability, defined with ARV showed positive correlation with left ventricular mass index ( $\mathrm{p}<0.05, \mathrm{r}=0.4442$, CI: 0.1732 to 0.6527 , versus 0.0533 to 0.668 in controls) (Figures 2 and 3). When BPV was defined with 24-hour systolic standard deviation, we found no relationship with LVMI in the elderly group ( $p=0.45, r=0.115$, CI: 0.1849 to 0.3953 ). Same results were obtained in the middle-aged control group ( $p=0.08, r=0.31$, CI:0.04745 to 0.6089 ). In elderly subjects increased 24-hour systolic BPV was positively correlated to increase in pulse pressure (PP) (Figure 4), as a marker of arterial stiffness ( $p=0.02, r=0.3275, \mathrm{CI}$ : 0.03753 to 0.5666 ). In the middle-aged group no correlation was found between 24-hour systolic BPV and PP ( $\mathrm{p}=0.16, \mathrm{r}=0.2631$, CI: 0.1074 to 0.5695 ). In the elderly group, 24-hour systolic BPV (ARV) was positively associated with morning BP surge (Figure 5) as well as with an increase in serum total cholesterol and triglyceride levels $(\mathrm{p}<0.05)$. No correlation was found between 24-hour systolic BPV and morning surge in the two groups $(\mathrm{p}>0.05)$. 
Table 1

Demographic, biohumoral, ABPM, BP variability and target organ damage data in elderly and middle-aged control group

\begin{tabular}{|c|c|c|c|}
\hline & Old & Middle-aged & p-value \\
\hline Gender, M/F & $14 / 31$ & $15 / 15$ & 0.16 \\
\hline Age, year & $70.89 \pm 1.064$ & $49.73 \pm 1.49$ & $<0.0001$ \\
\hline BMI, $\mathrm{g} / \mathrm{m}^{2}$ & $30.32 \pm 0.7$ & $30.39 \pm 1.2$ & 0.95 \\
\hline Total cholesterol, mg/dl & $205.5 \pm 7.79$ & $194.7 \pm 11.13$ & 0.4 \\
\hline Triglycerides, mg/dl & $164.9 \pm 7.99$ & $174.8 \pm 14$ & 0.5 \\
\hline Serum creatinine, mg/dl & $0.98 \pm 0.32$ & $1.09 \pm 0.63$ & 0.23 \\
\hline Blood glucose, mg/dl & $111.3 \pm 4.8$ & $107.4 \pm 4.26$ & 0.57 \\
\hline $\mathrm{MDRD}, \mathrm{ml} / \mathrm{min} / 1.73 \mathrm{~m}^{2}$ ) & $66.42 \pm 3.66$ & $80.03 \pm 5.14$ & 0.03 \\
\hline LVMI, $\mathrm{g} / \mathrm{m}^{2}$ & $125.4 \pm 3.74$ & $108.5 \pm 6.46$ & 0.01 \\
\hline ACEI & 39 & 16 & 0.003 \\
\hline ARBs & 2 & 2 & 0.67 \\
\hline Ca channel blocker & 25 & 11 & 0.17 \\
\hline Beta blocker & 21 & 15 & 0.96 \\
\hline Diuretic & 15 & 15 & 0.22 \\
\hline Central & 3 & 3 & 0.93 \\
\hline Statin & 10 & 1 & 0.053 \\
\hline 24-hour mean systolic BP, mmHg & $140.4 \pm 1.99$ & $133.0 \pm 1.94$ & 0.01 \\
\hline 24-hour mean diastolic $\mathrm{BP}, \mathrm{mmHg}$ & $71.64 \pm 1.22$ & $81.50 \pm 1.68$ & $<0.0001$ \\
\hline Daytime systolic BP, mmHg & $142.6 \pm 2.16$ & $137.8 \pm 2.50$ & 0.16 \\
\hline Daytime diastolic BP, mmHg & $74.07 \pm 1.3$ & $84.87 \pm 2.1$ & $<0.0001$ \\
\hline Nighttime systolic BP, mmHg & $133.9 \pm 2.18$ & $128.3 \pm 2.4$ & 0.09 \\
\hline Nighttime diastolic BP, $\mathrm{mmHg}$ & $66.49 \pm 1.26$ & $75.13 \pm 1.49$ & $<0.0001$ \\
\hline SD of average $24-\mathrm{h}$ BP & $15.18 \pm 0.46$ & $13.23 \pm 0.63$ & 0.01 \\
\hline Pulse pressure, $\mathrm{mmHg}$ & $65.51 \pm 2.18$ & $51.73 \pm 1.67$ & $<0.0001$ \\
\hline Dipper/non-dipper & $10 / 35$ & $10 / 20$ & 0.42 \\
\hline Morning surge, $\mathrm{mmHg}$ & $22.78 \pm 1.35$ & $18.33 \pm 1.59$ & 0.038 \\
\hline Daytime systolic ARV, $\mathrm{mmHg}$ & $11.8 \pm 0.45$ & $10.38 \pm 0.55$ & 0.05 \\
\hline Daytime diastolic ARV, $\mathrm{mmHg}$ & $7.53 \pm 0.24$ & $7.6 \pm 0.47$ & 0.88 \\
\hline Nighttime systolic ARV, mmHg & $11.18 \pm 0.55$ & $11.12 \pm 0.66$ & 0.94 \\
\hline Nighttime diastolic ARV, mmHg & $7.17 \pm 0.41$ & $7.48 \pm 0.52$ & 0.64 \\
\hline 24-hour systolic ARV, mmHg & $12.17 \pm 0.38$ & $10.9 \pm 0.45$ & 0.03 \\
\hline 24-hour diastolic ARV, mmHg & $7.51 \pm 1.49$ & $7.83 \pm 2.62$ & 0.54 \\
\hline
\end{tabular}

Note: $\mathrm{M}=$ male, $\mathrm{F}=$ female, $\mathrm{BMI}=$ body mass index, $\mathrm{MDRD}=$ modification of diet in renal disease study, $\mathrm{LVMI}=$ left ventricular mass index, $\mathrm{ACEI}=$ angiotensin-converting-enzyme inhibitor, $\mathrm{ARBs}=$ angiotensin receptor blockers, $\mathrm{BP}=$ blood pressure, $\mathrm{SD}=$ standard deviation, $\mathrm{ARV}=$ average real variability.

\section{Discussion}

The prevalence of hypertension increases with age and is still one of the leading causes of cardiovascular events. Therefore, management of hypertension in the elderly must take into account the presence of cardiovascular risk factors, circadian blood pressure profile, as well as blood pressure variability assessment. Therefore, this study assessed the relationship between blood pressure variability, cardiovascular risk factors, and target organ damage in elderly hypertensive patients, and a control group, consisting of middle-aged hypertensive patients.

According to other studies that evaluated BPV, the 24-hour ARV was a better predictor of target organ damage, than the commonly used 24-hour systolic SD, because patients with different blood pressure profiles might have the same SD value, but different ARV values [17]. 


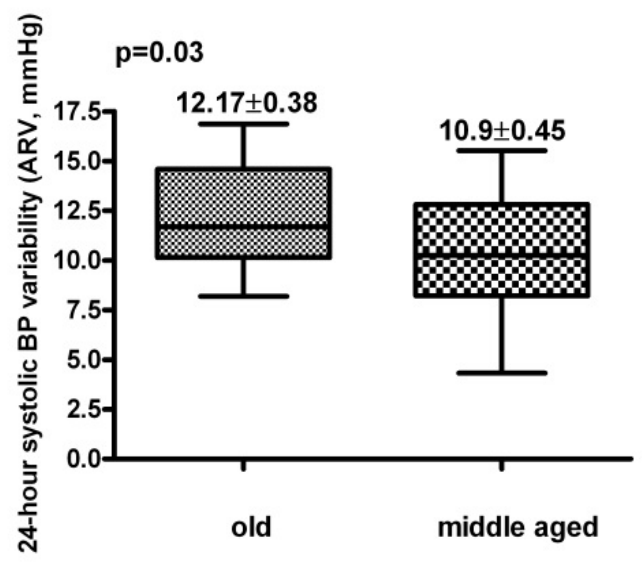

Fig. 1. Differences in 24-hour systolic BP variability (ARV) between elderly and middle-aged.

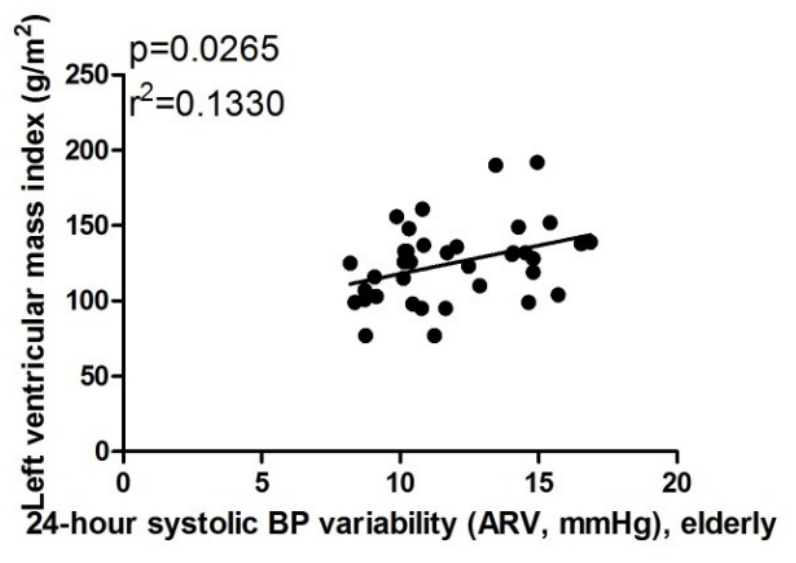

Fig. 2. Correlation between 24-hour systolic ARV and left ventricular mass index in elderly.

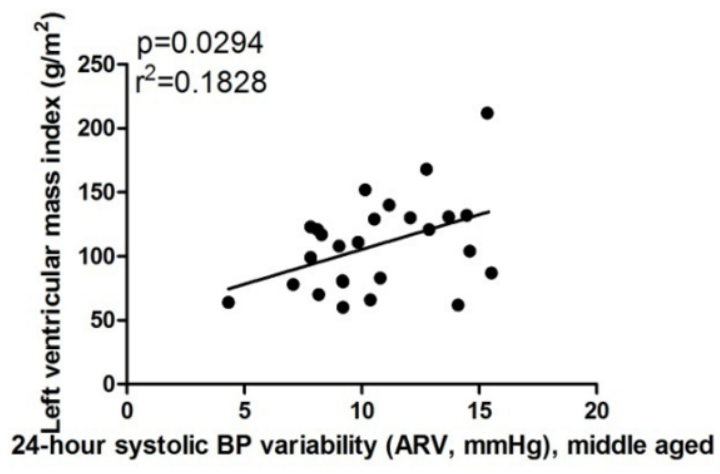

Fig. 3. Correlation between 24-hour systolic ARV and left ventricular mass index in middle-aged controls.

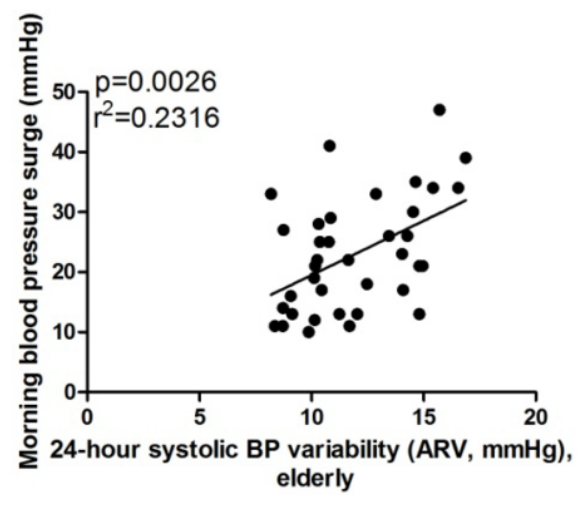

Fig. 4. Relationship between 24-hour systolic blood pressure variability and morning BP surge in elderly.

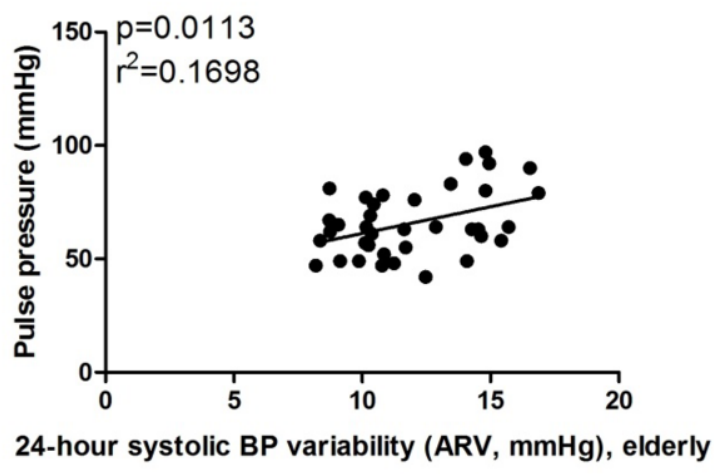

Fig. 5. Correlation between 24-hour systolic BP variability and pulse pressure in elderly. 
There exist lots of prospective studies on hypertensive patients, searching for association between blood pressure variability and cardiovascular outcomes, but this relation is yet not well established $[18,19]$. This might be due to low sample size, or the use of various indices of variability. Most studies still use the SD to define BPV, but these reflect only the dispersion of the data around the mean BP value [19]. Similar to other researchers, it was found that ARV might be a more specific measure of blood pressure variability than SD. It is less sensitive to the relative small sampling data, and the latest study made by Mena revealed that a number of 48 readings allow for accurate assessment of the relationship between ARV and cardiovascular risk, without loss of prognostic information [20]. Thus, it might be inefficient to assess daytime and nighttime BPV with the formula of ARV, due to insufficient sample size. In elderly hypertensive subjects showing increased systolic blood pressure, increased left ventricular mass, pulse pressure and morning surge, higher systolic ARV is also an independent predictor of cognitive decline, as shown in a Japanese study [21]. In elderly hypertensive subjects, increased left ventricular mass, as well as increased pulse pressure as a marker of arterial stiffness, is positively correlated to 24-hour systolic blood pressure variability (defined by ARV). This relationship was not observed in middle-aged group. This finding reflects the fact that blood pressure variability within 24-hour could be an additional cardiovascular risk factor. Elderly hypertensive patients are a vulnerable and inefficiently studied group, because behind increased systolic blood pressure, increased systolic BP variability is a characteristic of them, which should be taken into account by the selection of antihypertensive medication. The heart rate variability could be at least as important as the blood pressure variability because they are influencing each other. Perhaps the combined ambulatory monitoring, ECG and BP could lead to a more accurate treatment of the cardiovascular disease [24]. For quantifying BPV the selection of the variability index plays a crucial role. Nonetheless, further studies are needed to implement this index, and to establish a cut-off point or normal range.

\section{Conclusion}

The non-invasive 24-hour blood pressure monitoring offers the opportunity to evaluate read-to-read variability of BP. The average real variability index could be a more reliable index defining BPV because it is less sensitive to the low sampling size. In elderly patients, greater 24-hour systolic BP variability promotes an increase in left ventricular mass, and pulse pressure, therefore, drug therapy should be conducted more accurately, acting on reducing BP variability, and avoiding early target organ damage. The ABPM-derived BP variability index could be an early predictive marker of endorgan damage in hypertension. Results suggest that not only the mean blood pressure level but also blood pressure variability should be taken into account for the management of hypertension in elderly hypertensive patients.

\section{Acknowledgement}

This paper is supported by the Sectoral Operational Programme Human Resources Development (SOP HRD), financed from the European Social Fund and by the Romanian Government under the contract number POSDRU/159/1.5/S/133377. The work of L. Szilágyi was supported by UEFISCDI under project No. PD 28/05.08.2010. 
Special thanks to M. Kozlovszky and L. Kovács of Óbuda University of Budapest for support in data acquisition.

\section{References}

[1] A. Faini, G. Parati, M.D. Rienzo and P. Castiglioni, Night and day changes in heart rate and blood pressure fractal dimensions from 24-hour ambulatory blood pressure monitoring devices, Comput. Cardiol. 40 (2013), 475-478.

[2] P.M. Rothwell, S.C. Howard, E. Dolan, E. O'Brien, J.E. Dobson, B. Dahlöf et al., Prognostic significance of visit-tovisit variability, maximum systolic blood pressure, and episodic hypertension, Lancet 375 (2010), 895-905.

[3] G. Mancia, Short- and long-term blood pressure variability: Present and future, Hypertension 60 (2012), 512-517.

[4] A. Tatasciore, G. Renda, M. Zimarino, M. Soccio, G. Bilo, G. Parati et al., Awake systolic blood pressure variability correlates with target-organ damage in hypertensive subjects, Hypertension 50 (2007), 325-332.

[5] A.O. Ciobanu, C.L. Gherghinescu, R. Dulgheru, S. Magda, R. Dragoi Galrinho, M. Florescu et al., The impact of blood pressure variability on subclinical ventricular, renal and vascular dysfunction, in patients with hypertension and diabetes, Maedica (Buchar) 8 (2013), 129-136.

[6] N. Ozkayar, B. Altun, T. Yildirim, R. Yilmaz, F. Dede, G. Arik et al., Blood pressure measurements, blood pressure variability and endothelial function in renal transplant recipients, Clin. Exp. Hypertens, online 2013. doi:10.3109/10641963.2013.827706.

[7] G. Parati, J.E. Ochoa and G. Bilo, Blood pressure variability, cardiovascular risk, and risk for renal disease progression, Curr. Hypertens. Rep. 14 (2012), 421-431.

[8] V. Convanico, L.D. Caprio, C. Vigorito, L. Forgione, C. Tedeschi, P. Guarini et al., Differences in blood pressure profile between young and elderly hypertensive patients, J. Hum. Hypertens. 4 (1990), 405-409.

[9] M. Eto, M. Akishita, K. Kozaki, T. Watanabe, S. Kim, M. Hashimoto et al., Impact of blood pressure variability on cardiovascular events in elderly patients with hypertension, Hypertens. Res. 28 (2005), 1-7.

[10] J.I.M. Drayer, M.A. Weber, J.L. De Young and F.A. Wyle, Circadian blood pressure patterns in ambulatory hypertensive patients, effects of age, Am. J. Med. 73 (1982), 493-499.

[11] K. Kario and W.B. White, Early morning hypertension: What does it contribute to overall cardiovascular risk assessment? J. Am. Soc. Hypertens. 2 (2008), 397-402.

[12] L. Mena, S. Pintos, N.V. Queipo, J.A. Aizpúrua, G. Maestre and T. Sulbarán, A reliable index for the prognostic significance of blood pressure variability, J. Hypertens. 23 (2005), 505-511.

[13] R. Casadei, G. Parati, G. Pomidossi, A. Groppelli, S. Trazzi, M.D. Rienzo and G. Mancia, 24-hour blood pressure monitoring: Evaluation of Spacelabs 5300 monitor by comparison with intra-arterial blood pressure recording in ambulant subjects, J. Hypertens. 6 (1988), 797-803.

[14] G. Mancia, R. Fagard. K. Narkiewicz, J. Redón, A. Zanchetti, M. Böhm et al., 2013 ESH/ESC Guidelines for the management of arterial hypertension: The task force for the management of arterial hypertension of the European Society of Hypertension (ESH) and of the European Society of Cardiology (ESC), J. Hypertens. 31 (2013), 1281-1357.

[15] A.S. Levey, L.A. Stevens, C.H. Schmid, Y.L. Zhang, A.F. Castro 3rd, H.I. Feldman, CKD-EPI (Chronic Kidney Disease Epidemiology Collaboration) et al., A new equation to estimate glomerular filtration rate, Ann. Intern. Med., 150 (2009), 604-612.

[16] R.B. Devereux, D.R. Alonso, E.M. Lutas et al., Echocardiographic assessment of left ventricular hypertrophy: Comparison to necropsy findings, Am. J. Cardiol. 57 (1986), 450-458.

[17] T.W. Hansen, L. Thijs, Y. Li, J. Boggia, M. Kikuya and K. Björklund-Bodegård, Prognostic value of reading-to-reading blood pressure variability over 24 hours in 8938 subjects from 11 populations, Hypertension 55 (2010), 1049-1057.

[18] K. Eguchi, J. Ishikawa, S. Hoshide, T.G. Pickering, J.E. Schwartz, K. Shimada and K. Kario, Nighttime blood pressure variability is a strong predictor for cardiovascular events in patients with type 2 diabetes, Am. J. Hypertens. 2 (2009), 46-51.

[19] S.D. Pierdomenico, M.D. Nicola, A.L. Esposito, R.D. Mascio, E. Ballone, D. Lapenna et al., Prognostic value of different indices of blood pressure variability in hypertensive patients, Am. J. Hypertens. 22 (2009), 842-847.

[20] L.J. Mena, G.E. Maestre, T.W. Hansen, L. Thijs, Y. Liu, J. Boggia et al., How many measurements are needed to estimate blood pressure variability without loss of prognostic information? Am. J. Hypertens. 27 (2014), 46-66.

[21] Y. Yoshitaka, W. Manabu, S. Hidenori, N. Hikaru, K. Shingo, T. Yoshimi et al., Impact of ambulatory blood pressure variability on cerebral small vessel disease progression and cognitive decline in community-based elderly Japanese, Am. J. Hypertens., online 2014. doi: 10.1093/ajh/hpu045. 